\title{
Displacer Synthesis
}

Propylsulfonation of Trehalose Derivatives (Scheme1). TH(PSNa) $)_{8}$ was synthesized by the following procedure. To a suspension of $\mathrm{NaH}(95 \%, 1.76 \mathrm{~g}, 70.1 \mathrm{mmols})$ in anhydrous DMSO was added dropwise trehalose $(2.5 \mathrm{~g}, 7.3 \mathrm{mmols})$ through a dropping funnel under nitrogen with magnetic stirring. The reaction mixture was heated at $50{ }^{\circ} \mathrm{C}$ for $2 \mathrm{hr}$. Into the suspension 1,3-propansultone (10.0 g, 82.0 mmols) was added dropwise. The reaction mixture was heated at $50{ }^{\circ} \mathrm{C}$ for $12 \mathrm{hr}$. and $80{ }^{\circ} \mathrm{C}$ for $12 \mathrm{hr}$. The mixture was cooled to room temperature and filtered through a fritted filter to remove any precipitate. An ethanol/water mixture (9/1) was added slowly with stirring to cause precipitation. The solid was collected, washed with a mixture of ethanol/water (9/1), and dried at $50{ }^{\circ} \mathrm{C}$ under vacuum. The product was purified further by precipitation from DMF/Water (6/1), washing with DMF, and drying under vacuum. Finally, the product was stirred in boiling methanol for $1 \mathrm{hr}$., filtered and dried under vacuum at $60^{\circ} \mathrm{C}$ to give 5.9 $\mathrm{g}$ of the desired product in 54\% yield. ${ }^{1} \mathrm{H}$ NMR $\left(500 \mathrm{MHz}, \mathrm{DMSO}-\mathrm{d}_{6}\right) \delta 4.99(\mathrm{~d}, 2 \mathrm{H}$, anomeric $\mathrm{H})$, $3.72(\mathrm{~m}, 6 \mathrm{H}), 3.60(\mathrm{~m}, 4 \mathrm{H}), 3.45(\mathrm{~m}, 10 \mathrm{H}), 3.34(\mathrm{~m}, 4 \mathrm{H}), 3.18(\mathrm{~m}, 4 \mathrm{H}), 2.56(\mathrm{~m}, 16 \mathrm{H}), 1.80(\mathrm{~m}$, 16H). ${ }^{13} \mathrm{C}$ NMR $\left(125 \mathrm{MHz}, \mathrm{DMSO}-\mathrm{d}_{6}\right) \delta 92.48,80.83,79.52,77.18,71.91,71.46,70.19,69.85$, $69.38,68.66,48.59,48.51,48.42,48.16,26.39,26.18,25.83,25.62$.

Enzyme-Catalyzed Synthesis of Di-substituted Trehalose Esters (Scheme 1). The reaction was performed with Novozym-435 $(10 \mathrm{mg} / \mathrm{mL})$ at $50{ }^{\circ} \mathrm{C}$ using $0.1 \mathrm{M}$ trehalose and $0.4 \mathrm{M}$ of vinylbutylate, vinyl myristate, or vinyl benzoate in acetone for 2 days as described in the literature ${ }^{1}$. The reaction progress was checked by thin layer chromatography on silica with an eluent consisting of ethylacetate/methanol/water (16/6/1) and detection by charring with $10 \% \mathrm{H}_{2} \mathrm{SO}_{4}$ in ethanol on a hot plate. After the reaction was completed, the solid pattern was collected by filtration with a fritted glass filter. To the filtrate was added silica gel and then the solvent was evaporated with a rotary 
evaporator. The adsorbed product on silica gel was purified by column chromatography using a mixture of ethylacetate/methanol $(8 / 2 \sim 7 / 3)$ as eluent. The isolated yields of disubstituted trehalose derivatives were in the range of $10 \sim 25 \%$. DBuTH : yield $0.42 \mathrm{~g}, 0.87 \mathrm{mmol}, 15 \%$; ${ }^{1} \mathrm{H}$ NMR (500 MHz, DMSO-d $\left.{ }_{6}\right) \delta 5.08(\mathrm{~d}, 2 \mathrm{H}), 4.91(\mathrm{~d}, 2 \mathrm{H}), 4.80(\mathrm{~d}, 4 \mathrm{H}), 4.23(\mathrm{dd}, 2 \mathrm{H}), 4.04(\mathrm{~m}, 2 \mathrm{H}), 3.89(\mathrm{~m}$, 2H), $3.51(\mathrm{~m}, 2 \mathrm{H}), 3.23(\mathrm{~m}, 2 \mathrm{H}), 3.12(\mathrm{~m}, 2 \mathrm{H}), 2.25(\mathrm{t}, 4 \mathrm{H}), 1.53(\mathrm{st}, 4 \mathrm{H}), 0.86(\mathrm{t}, 6 \mathrm{H})$. DMyTH: yield $0.8 \mathrm{~g}, 1.04 \mathrm{mmol}, 25 \% ;{ }^{1} \mathrm{H}$ NMR (500 MHz, DMSO-d 6 ) $\delta 5.06(\mathrm{~d}, 2 \mathrm{H}), 4.88(\mathrm{~d}, 2 \mathrm{H}), 4.80(\mathrm{~d}$, 2H), $4.77(\mathrm{~d}, 2 \mathrm{H}), 4.20(\mathrm{~d}, 2 \mathrm{H}), 4.02(\mathrm{~m}, 2 \mathrm{H}), 3.85(\mathrm{~m}, 2 \mathrm{H}), 3.52(\mathrm{~m}, 2 \mathrm{H}), 3.23(\mathrm{~m}, 2 \mathrm{H}), 3.10(\mathrm{~m}$, 2H), $2.26(\mathrm{t}, 4 \mathrm{H}), 1.49(\mathrm{~m}, 4 \mathrm{H}), 1.22$ (br.s, 40H), $0.84(\mathrm{t}, 6 \mathrm{H}) .{ }^{13} \mathrm{C}$ NMR $\left(125 \mathrm{MHz}, \mathrm{D}_{2} \mathrm{O}\right)$ $\delta 174.16,88.35,74.24,72.56,72.00,66.15,60.72,32.02,29.93,27.69$ (overlapping peaks), 27.38, 27.06, 22.70, 20.63, 11.95. DBzTH: yield $0.3 \mathrm{~g}, 0.54 \mathrm{mmol}, 10 \%$; ${ }^{1} \mathrm{H}$ NMR (500 MHz, DMSO-d $\left._{6}\right) \delta 7.96(\mathrm{~m}, 4 \mathrm{H}), 7.64(\mathrm{t}, 2 \mathrm{H}), 7.52(\mathrm{~m}, 4 \mathrm{H}), 5.22(\mathrm{~d}, 2 \mathrm{H}), 4.97(\mathrm{~m}, 4 \mathrm{H}), 4.88(\mathrm{~d}, 2 \mathrm{H}), 4.41$ $(\mathrm{dd}, 2 \mathrm{H}), 4.32(\mathrm{~m}, 2 \mathrm{H}), 4.11(\mathrm{~m}, 2 \mathrm{H}), 3.60(\mathrm{~m}, 2 \mathrm{H}), 3.35(\mathrm{~m}, 2 \mathrm{H}), 3.27(\mathrm{~m}, 2 \mathrm{H}) .{ }^{13} \mathrm{C}$ NMR $(125$ MHz, DMSO-d 6 ) $\delta 165.69,133.30,129.80,129.18,128.77,93.87,72.84,71.49,70.14,69.82,64.07$.

Enzyme-Catalyzed Synthesis of Mono-substituted Trehalose Esters (Scheme 1). The reaction was conducted with ProteaseCL-15 at room temperature in DMF containing 0.125M of trehalose, $0.5 \mathrm{M}$ of vinyl myristate, or vinyl benzoate, and $2.8 \mathrm{M}$ of water for 12 days according to a similar literature method ${ }^{2}$. The reaction progress was confirmed by thin layer chromatography on silica with an eluent consisting of ethylacetate/methanol/water (16/7/1) and detection by charring with $10 \% \mathrm{H}_{2} \mathrm{SO}_{4}$ in ethanol on a hot plate. After the reaction was completed, solid was removed by filtration with a fritted filter. The solvent was evaporated from the filtrate by simple vacuum distillation. Methanol was added to the residue until the solid dissolved. Silica gel was added and the solvent was evaporated with a rotary evaporator. The product adsorbed on silica gel was purified by column chromatography using a mixture of ethylacetate/methanol (8/2 5/5) as eluent. Only in the 
case of MBzTH, a 16/7/1 ratio of ethylacetate/methanol/water was used as eluent. The isolated yields of mono-substituted trehalose derivatives were in the range of $10 \sim 30 \%$. MMyTH: yield 1.05 g, 1.9 mmol, 30\%; ${ }^{1} \mathrm{H}$ NMR (500 MHz, DMSO-d 6 ) $\delta 5.05(\mathrm{~d}, 1 \mathrm{H}), 4.87(\mathrm{~d}, 1 \mathrm{H}), 4.84(\mathrm{~d}, 1 \mathrm{H}), 4.82$ $(\mathrm{d}, 1 \mathrm{H}), 4.77(\mathrm{~d}, 2 \mathrm{H}), 4.67(\mathrm{~d}, 2 \mathrm{H}), 4.34(\mathrm{t}, 1 \mathrm{H}), 4.20(\mathrm{~d}, 1 \mathrm{H}), 4.01(\mathrm{~m}, 1 \mathrm{H}), 3.87(\mathrm{~m}, 1 \mathrm{H}), 3.64(\mathrm{~m}$, 1H), $3.53(\mathrm{~m}, 3 \mathrm{H}), 3.45(\mathrm{~m}, 1 \mathrm{H}), 3.23(\mathrm{~m}, 2 \mathrm{H}), 3.11(\mathrm{~m}, 2 \mathrm{H}), 2.25(\mathrm{t}, 2 \mathrm{H}), 1.48(\mathrm{t}, 2 \mathrm{H}), 1.22$ (br.s, 20H), $0.83(\mathrm{t}, 3 \mathrm{H}) .{ }^{13} \mathrm{C}$ NMR (125 MHz, DMSO-d 6 ) $\delta 172.83,93.32,93.22,72.82,72.77,72.58$, $71.56,71.45,70.09,69.63,63.08,60.76,33.51,31.29,29.07,29.04,29.01,28.90,28.74,28.71$, 28.46, 24.44, 22.08, 13.94. MBzTH: yield $0.3 \mathrm{~g}, 0.67 \mathrm{mmol}, 10 \%$; ${ }^{1} \mathrm{H}$ NMR (500 MHz, DMSO-d 6 ) $\delta 7.97(\mathrm{~d}, 2 \mathrm{H}), 7.65(\mathrm{t}, 1 \mathrm{H}), 7.53(\mathrm{~d}, 2 \mathrm{H}), 5.20(\mathrm{~d}, 1 \mathrm{H}), 4.95(\mathrm{~m}, 1 \mathrm{H}), 4.87-4.80(\mathrm{~m}, 4 \mathrm{H}), 4.74(\mathrm{~d}$, 1H), $4.42(\mathrm{~d}, 1 \mathrm{H}), 4.35(\mathrm{~m} \mathrm{1H}), 4.10(\mathrm{~m}, 1 \mathrm{H}), 3.65(\mathrm{~m}, 1 \mathrm{H}), 3.56(\mathrm{~m}, 3 \mathrm{H}), 3.46(\mathrm{~m}, 1 \mathrm{H}), 3.36-3.24$ (m, 5H), $3.13(\mathrm{~m}, 1 \mathrm{H}) .{ }^{13} \mathrm{C}$ NMR $\left(125 \mathrm{MHz}, \mathrm{DMSO}-\mathrm{d}_{6}\right) \delta 165.78,133.41,129.86,129.24,128.86$, 93.42, 93.37, 72.92(overlap of 1C), 72.66, 71.64, 71.60, 70.29, 70.13, 69.76, 64.18, 60.79. MBuTH: yield 0.2 g, 0.48 mmol, 14\%; ${ }^{1} \mathrm{H}$ NMR (500 MHz, DMSO-d $\left.{ }_{6}\right) \delta 5.05$ (d, 1H), 4.87 (d, 1H), $4.82(\mathrm{dd}, 2 \mathrm{H}), 4.77(\mathrm{~m}, 2 \mathrm{H}), 4.68(\mathrm{~d}, 2 \mathrm{H}), 4.35(\mathrm{t}, 1 \mathrm{H}), 4.21(\mathrm{~d}, 1 \mathrm{H}), 4.02(\mathrm{dd}, 1 \mathrm{H}), 3.88(\mathrm{~m}$, 1H), 3.63(m, 1H), 3.53(m, 3H), $3.46(\mathrm{~m}, 1 \mathrm{H}), 3.24(\mathrm{~m}, 2 \mathrm{H}), 3.14(\mathrm{~m}, 2 \mathrm{H}), 2.25(\mathrm{t}, 2 \mathrm{H}), 1.51(\mathrm{st}, 2 \mathrm{H})$, $0.87(\mathrm{t}, 3 \mathrm{H}) .{ }^{13} \mathrm{C}$ NMR $\left(125 \mathrm{MHz}, \mathrm{DMSO}-\mathrm{d}_{6}\right) \delta 172.75,93.34,93.24,72.83,72.76,72.60,71.55$, 71.44, 70.11, 70.03, 69.66, 63.10, 60.71, 35.42, 17.95, 13.47.

Sulfonation of Trehalose Derivatives (Scheme 1) ${ }^{3}$. Sulfur trioxide-trimethylamine (4 equivalent per hydroxyl group) the trehalose derivative (MBuTH, MMyTH, MBzTH, DBuTH, DMyTH, and DBzTH) and DMF (20 30mL) were placed in a round bottom flask. The reaction mixture was degassed under vacuum with magnetic stirring for $10 \mathrm{~min}$. After nitrogen was admitted into the flask, the reaction mixture was heated at $60{ }^{\circ} \mathrm{C}$ for 1 day. Only in the case of neomycin was the reaction performed for 4 days at a temperature of $75{ }^{\circ} \mathrm{C}$. The reaction mixture was cooled to room 
temperature. The solvent was removed by simple vacuum distillation while increasing the temperature to $40{ }^{\circ} \mathrm{C}$. To the residue was added $10 \%$ aqueous $\mathrm{Na}_{2} \mathrm{CO}_{3}$ solution with stirring in an ice-water bath. The solution was stirred for $4 \mathrm{hr}$. at room temperature. As the reaction proceeded, the by-products precipitated. After the reaction mixture was filtered, acetone was slowly added to the obtained solution to precipitate the product. In case of $\mathbf{M M y T H}\left(\mathbf{S O}_{3} \mathbf{N a}\right)_{7}$ and $\mathbf{D M y T H}\left(\mathbf{S O}_{3} \mathbf{N a}\right)_{6}$, methanol was used instead of acetone for the precipitation. The white solid was dried under vacuum at room temperature. To purify further each product, it was dialyzed for 1 2 days in distilled water using a dialysis bag with a molecular weight cut off equal to 100 . The product in the bag was precipitated from acetone or methanol and dried under vacuum at $50{ }^{\circ} \mathrm{C}$ overnight. The isolated yields of the products were in the range of $30 \sim 65 \%$. MMyTH(SO $\left.\mathbf{S O}_{3} \mathbf{N a}\right)_{7}$ : yield $0.8 \mathrm{~g}, 0.63 \mathrm{mmol}$, $65 \% ;{ }^{1} \mathrm{H}$ NMR $\left(500 \mathrm{MHz}, \mathrm{D}_{2} \mathrm{O}\right) \delta 5.58(\mathrm{dd}, 2 \mathrm{H}), 4.85(\mathrm{ddd}, 2 \mathrm{H}), 4.53-4.35(\mathrm{~m}, 10 \mathrm{H}), 2.44(\mathrm{t}, 2 \mathrm{H})$, 1.62 (qt, 2H), 1.29 (br.s, 20H), 0.87 (t, 3H). $\left.{ }^{13} \mathrm{C} \mathrm{NMR} \mathrm{(125} \mathrm{MHz,} \mathrm{D}_{2} \mathrm{O}\right) \delta 177.06,92.49$ (overlap of 1C), 76.05, 75.95, 74.72, 74.54, 73.82, 68.82, 68.61, 66.02, 62.57, 33.90, 31.53, 29.09(overlap of 2C), 28.53 (overplap of 3C), 24.38, 22.37, 13.74. ESI for $\mathrm{C}_{26} \mathrm{H}_{41} \mathrm{O}_{33} \mathrm{~S}_{4} \mathrm{Na}_{7}$ [M-Na] calcd. 1242.90, found 1242.70. MBzTH(SO $\left.\mathbf{S O}_{3} \mathbf{N a}\right)_{7}$ : yield $0.6 \mathrm{~g}, 0.52 \mathrm{mmol}, 61 \% ;{ }^{1} \mathrm{H}$ NMR $\left(500 \mathrm{MHz}, \mathrm{D}_{2} \mathrm{O}\right) \delta 8.12$ $(\mathrm{d}, 2 \mathrm{H}), 7.72(\mathrm{t}, 1 \mathrm{H}), 7.59(\mathrm{t}, 2 \mathrm{H}), 5.62(\mathrm{dd}, 2 \mathrm{H}), 4.89(\mathrm{~m}, 2 \mathrm{H}), 4.74(\mathrm{~m}, 2 \mathrm{H}), 4.50-4.48(\mathrm{~m}, 10 \mathrm{H})$. ${ }^{13} \mathrm{C}$ NMR $\left(125 \mathrm{MHz}, \mathrm{D}_{2} \mathrm{O}\right) \delta 165.73,134.03,129.78,129.43,128.91,92.69,93.00,76.05,75.75$, 74.81, 74.60, 73.93, 73.73, 68.82, 68.77, 66.08, 63.18. ESI for $\mathrm{C}_{19} \mathrm{H}_{19} \mathrm{O}_{33} \mathrm{~S}_{7} \mathrm{Na}_{7}[\mathrm{M}-\mathrm{Na}]^{-}$calcd. 1136.72, found 1136.60. DBuTH(SO $\mathbf{3} \mathbf{N a})_{6}$ : yield $0.15 \mathrm{~g}, 0.14 \mathrm{mmol}, 30 \% ;{ }^{1} \mathrm{H}$ NMR (500 MHz, $\left.\mathrm{D}_{2} \mathrm{O}\right) \delta 5.52(\mathrm{~d}, 2 \mathrm{H}), 4.50-4.35(\mathrm{~m}, 12 \mathrm{H}), 2.38(\mathrm{t}, 4 \mathrm{H}), 1.60(\mathrm{st}, 4 \mathrm{H}), 0.89(\mathrm{t}, 6 \mathrm{H}) .{ }^{13} \mathrm{C}$ NMR $(125$ $\left.\mathrm{MHz}, \mathrm{D}_{2} \mathrm{O}\right) \delta 176.95,92.98,75.78,74.74,73.85,68.73,62.52,35.84,18.01,13.06$. ESI for $\mathrm{C}_{20} \mathrm{H}_{28} \mathrm{O}_{31} \mathrm{~S}_{6} \mathrm{Na}_{6}[\mathrm{M}+\mathrm{Na}]^{+}$calcd. 1116.8, found 1116.7. DBzTH(SO $\left.\mathbf{S}_{3} \mathbf{N a}\right)_{6}:$ yield $0.57 \mathrm{~g}, 0.49$ mmol, 64\%; ${ }^{1} \mathrm{H}$ NMR (500 MHz, $\left.\mathrm{D}_{2} \mathrm{O}\right) \delta 8.08(\mathrm{~d}, 4 \mathrm{H}), 8.06(\mathrm{t}, 2 \mathrm{H}), 7.54(\mathrm{t}, 4 \mathrm{H}), 5.62(\mathrm{~d}, 2 \mathrm{H}), 4.93$ $(\mathrm{t}, 2 \mathrm{H}), 4.71(\mathrm{~m}, 4 \mathrm{H}), 4.61-4.54(\mathrm{~m}, 6 \mathrm{H}) .{ }^{13} \mathrm{C} \mathrm{NMR}\left(125 \mathrm{MHz}, \mathrm{D}_{2} \mathrm{O}\right) \delta 168.63,134.02,129.77$, 
129.39, 128.89, 92.87, 75.92, 74.83, 73.95, 68.89, 63.14. ESI for $\mathrm{C}_{26} \mathrm{H}_{24} \mathrm{O}_{31} \mathrm{~S}_{6} \mathrm{Na}_{6}[\mathrm{M}+\mathrm{Na}]^{+}$calcd.

1184.79, found 1184.50. DMyTH(SO $\left.\mathbf{S O}_{3} \mathbf{N a}\right)_{6}$ : yield $0.6 \mathrm{~g}, 0.43 \mathrm{mmol}, 58 \% ;{ }^{1} \mathrm{H} \mathrm{NMR}\left(500 \mathrm{MHz}, \mathrm{D}_{2} \mathrm{O}\right)$

$\delta 5.53$ (br.s, 2H), 4.86 (t, 2H), 4.49-4.25 (m, 10H), 2.42 (br.s, 4H), 1.62 (br.s, 4H), 1.27 (br.s, 43H),

$0.90(\mathrm{t}, 3) . \quad{ }^{13} \mathrm{C}$ NMR $\left(125 \mathrm{MHz}, \mathrm{D}_{2} \mathrm{O}\right) \delta 176.69,91.17,77.00,75.51,75.51,75.22,69.21,63.55$,

$34.73,32.36,30.12,30.07,29.95$ (overlap of 1C), 29.75, 29.70, 29.56, 25.28, 23.05, 14.35. ESI

for $\mathrm{C}_{40} \mathrm{H}_{68} \mathrm{O}_{31} \mathrm{~S}_{6} \mathrm{Na}_{6} \quad[\mathrm{M}+\mathrm{Na}]^{+}$calcd. 1397.14, found 1397.10. $\mathbf{T H}\left(\mathbf{S O} \mathbf{O}_{3} \mathbf{N a}\right)_{7}$ : This product is synthesized from hydrolysis of $\mathrm{MBuTH}\left(\mathrm{SO}_{3} \mathrm{Na}\right)_{7}$ with heating at $50{ }^{\circ} \mathrm{C}$ for $4 \mathrm{hr}$. in the presence of $10 \% \mathrm{Na}_{2} \mathrm{CO}_{3}$ aqueous solution. The purification step is the same as above. Yield $0.12 \mathrm{~g}, 0.11$ mmol, 31\%; ${ }^{1} \mathrm{H}$ NMR (500 MHz, $\left.\mathrm{D}_{2} \mathrm{O}\right) \delta 5.54(\mathrm{dd}, 2 \mathrm{H}), 4.43-4.29(\mathrm{~m}, 10 \mathrm{H}), 4.12(\mathrm{~d}, 1 \mathrm{H}), 3.92(\mathrm{dd}$, $1 \mathrm{H}), 3.83(\mathrm{~d}, 1 \mathrm{H}) .{ }^{13} \mathrm{C}$ NMR $\left(125 \mathrm{MHz}, \mathrm{D}_{2} \mathrm{O}\right) \delta 92.57,92.38,75.97,75.90,74.89,74.59,73.86$, 73.80, 70.84, 68.78, 66.10, 59.93. ESI for $\mathrm{C}_{12} \mathrm{H}_{15} \mathrm{O}_{32} \mathrm{~S}_{7} \mathrm{Na}_{7}[\mathrm{M}+\mathrm{Na}]^{+}$calcd. 1078.68, found 1078.50. $\mathbf{T H}\left(\mathbf{S O}_{\mathbf{3}} \mathbf{N a}\right)_{\mathbf{8}}{ }^{4}$ : Trehalose $(0.33 \mathrm{~g}, 0.96 \mathrm{mmol})$, sulfur trioxide pyridine complex $(1.84 \mathrm{~g}$, $11.52 \mathrm{mmols})$, pyridine $(20 \mathrm{~mL})$ were placed in a 2-neck a round bottom flask under nitrogen. The reaction mixture was heated to $65{ }^{\circ} \mathrm{C}$ and maintained at that temperature overnight. After the reaction was finished, the product separated as a yellowish oil. While the reaction mixture was still hot, pyridine was decanted and the residue dissolved in distilled water $(5 \mathrm{~mL})$. The solution was cooled in an ice bath and the $\mathrm{pH}$ was adjusted to $8 \sim 9$ by slowly adding of $20 \%$ aqueous $\mathrm{NaOH}$ solution with stirring. The product was precipitated by the slow addition of methanol. The white solid was filtered and washed with a 1/1 solution of water/methanol followed by pure methanol. The filter cake was dried at $50{ }^{\circ} \mathrm{C}$ under vacuum, overnight. The obtained product was dissolved in water, crystallized by the slow addition of methanol, washed with a 1/2 solution of water/methanol followed by pure methanol and dried at $50{ }^{\circ} \mathrm{C}$ under vacuum. To purify the product further, it was crystallized three additional times as above to give $0.45 \mathrm{~g}$ of $\mathbf{T H}\left(\mathbf{S O}_{\mathbf{3}} \mathbf{N a}\right)_{\mathbf{8}}$ in $40 \%$ yield. ${ }^{1} \mathrm{H} \mathrm{NMR}$ $\left(500 \mathrm{MHz}, \mathrm{D}_{2} \mathrm{O}\right) \delta 5.59(\mathrm{~d}, 2 \mathrm{H}$, anomeric $\mathrm{H}), 4.85(\mathrm{t}, 2 \mathrm{H}), 4.52-4.36(\mathrm{~m}, 10 \mathrm{H}) . \quad{ }^{13} \mathrm{C} \mathrm{NMR}(125 \mathrm{MHz}$, 
$\left.\mathrm{D}_{2} \mathrm{O}\right) \delta 92.52,76.33,74.82,74.19,69.11,66.38$. ESI for $\mathrm{C}_{12} \mathrm{H}_{14} \mathrm{O}_{35} \mathrm{~S}_{8} \mathrm{Na}_{8}[\mathrm{M}+\mathrm{Na}]^{+}$calcd. 1180.6, found 1180.5 .

Sulfonation and Alkylsufonation of Monosaccharides (Scheme 2). Sulfonation of PhGal was done according to the same procedure as that for the trehalose derivatives. Propylsulfonation or butylsulfonation of monosaccharides (MtGlu, OctGlu, HexGlu, DodGlu, PhGlu, PhGal and $\mathrm{NpBrGlu}$ ) was carried out by the reaction of 1,3-propane sultone or 1,4-butane sultone (1.5 equivalent per hydroxyl group) with the alkoxide generated by reaction with sodium hydride (1.2 equivalent per hydroxyl group) in DMSO (30 ml/g of monosaccharide) at room temperature. The reaction temperature was kept at $60-70{ }^{\circ} \mathrm{C}$ for propylsulfonation and $65-75{ }^{\circ} \mathrm{C}$ for butanesulfonation for 1 day. Purification of the sulfonated products was conducted by precipitation from ethanol and by washing the dried products in methanol with stirring. Finally, the resulting products were dried in a vacuum oven at $40{ }^{\circ} \mathrm{C}$ for one day to give isolated yields of $44 \%-80 \%$. MtGlu(PSNa) 4: yield 1.10 g, $1.42 \mathrm{mmol}, 80 \% ;{ }^{1} \mathrm{H}$ NMR(500 MHz, D $\left.2 \mathrm{O}\right) \delta 4.94$ (d, 1H), 3.92 $-3.88(\mathrm{~m}, 3 \mathrm{H}), 3.78-3.59(\mathrm{~m}, 9 \mathrm{H}), 3.43(\mathrm{~m}, 2 \mathrm{H}), 3.41(\mathrm{~s}, 3 \mathrm{H}), 3.01-2.96(\mathrm{~m}, 8 \mathrm{H}), 2.03(\mathrm{~m}, 8 \mathrm{H})$. ${ }^{13} \mathrm{C}$ NMR $\left(125 \mathrm{MHz}, \mathrm{D}_{2} \mathrm{O}\right) \delta 97.46,81.39,79.63,77.79,72.22,71.19,69.82,69.73,69.52,68.89$, 55.21, 48.30, 48.22, 48.16, 48.08, 25.38, 25.32, 25.13, 24.56. ESI for $\mathrm{C}_{19} \mathrm{H}_{34} \mathrm{O}_{18} \mathrm{~S}_{4} \mathrm{Na}_{4}$ [M-Na] calcd. 747.03, found 746.90. MtGlu(BuSNa) 4 : yield $0.82 \mathrm{~g}, 0.99 \mathrm{mmol}, 64 \%$; ${ }^{1} \mathrm{H}$ NMR(500 MHz, $\left.\mathrm{D}_{2} \mathrm{O}\right)$ $\delta \quad 4.96(\mathrm{~d}, 1 \mathrm{H}), 3.88-3.70(\mathrm{~m}, 3 \mathrm{H}), 3.75-3.61(\mathrm{~m}, 9 \mathrm{H}), 3.44(\mathrm{~m}, 3 \mathrm{H}), 3.43(\mathrm{~m}, 2 \mathrm{H}), 2.97(\mathrm{~m}, 8 \mathrm{H})$, $1.86-1.74(\mathrm{~m}, 16 \mathrm{H}) .{ }^{13} \mathrm{C} \mathrm{NMR}\left(125 \mathrm{MHz}, \mathrm{D}_{2} \mathrm{O}\right) \delta 97.56,81.32,79.62,77.08,73.44,73.16,71.05$, $70.97,69.59,68.87,55.26,50.97,50.89$ (overlap of $2 \mathrm{C}$ ), 28.62, 28.52, 28.29, 27.74, 21.28, 21.2 (overlap of 1C), 21.09. ESI for $\mathrm{C}_{23} \mathrm{H}_{42} \mathrm{O}_{18} \mathrm{~S}_{4} \mathrm{Na}_{4}[\mathrm{M}+\mathrm{Na}]^{+}$calcd. 849.07, found 849.00. OctGlu(PSNa $)_{4}$ : yield $0.75 \mathrm{~g}, 0.86 \mathrm{mmol}, 64 \% ;{ }^{1} \mathrm{H}$ NMR(500 MHz, D $\left.2 \mathrm{O}\right) \delta \quad 4.47(\mathrm{~d}, 1 \mathrm{H}), 3.96$ $3.69(\mathrm{~m}, 12 \mathrm{H}), 3.55(\mathrm{~m}, 1 \mathrm{H}), 3.48(\mathrm{~m}, 1 \mathrm{H}), 3.38(\mathrm{~m}, 1 \mathrm{H}), 3.17(\mathrm{t}, 1 \mathrm{H}), 3.01(\mathrm{~m}, 8 \mathrm{H}), 2.07(\mathrm{~m}, 8 \mathrm{H})$, 
1.66 (qnt, 2H), $1.40-1.31(\mathrm{~m}, 10 \mathrm{H}), 0.90(\mathrm{~m}, 3 \mathrm{H}) .{ }^{13} \mathrm{C} \mathrm{NMR}\left(125 \mathrm{MHz}, \mathrm{D}_{2} \mathrm{O}\right) \delta$ 102.51, 84.05, $81.76,78.12,73.72,72.31,71.82,71.74,71.14,69.77,69.16,48.38,48.23,48.13$ (overlap of 1C), $31.35,29.02,28.65,28.45,25.47,25.31$ (overlap of 2C), 24.60, 22.27, 13.72. ESI for $\mathrm{C}_{26} \mathrm{H}_{48} \mathrm{O}_{18} \mathrm{~S}_{4} \mathrm{Na}_{4}$ [M-Na] calcd. 845.14, found 845.00. OctGlu(BuSNa) ${ }_{4}$ : yield $0.63 \mathrm{~g}, 0.68 \mathrm{mmol}$, $53 \% ;{ }^{1} \mathrm{H} \mathrm{NMR}\left(500 \mathrm{MHz}, \mathrm{D}_{2} \mathrm{O}\right) \delta 4.44(\mathrm{~d}, 1 \mathrm{H}), 3.95-3.63(\mathrm{~m}, 12 \mathrm{H}), 3.53(\mathrm{~m}, 1 \mathrm{H}), 3.41(\mathrm{t}, 1 \mathrm{H})$, $3.31(\mathrm{t}, 1 \mathrm{H}), 3.13(\mathrm{t}, 1 \mathrm{H}), 2.95(\mathrm{~m}, 8 \mathrm{H}), 1.82(\mathrm{~m}, 16 \mathrm{H}), 1.63(\mathrm{qnt}, 2 \mathrm{H}), 1.39-1.29(\mathrm{~m}, 10 \mathrm{H}), 0.87$ (m, 3H). ${ }^{13} \mathrm{C}$ NMR $\left(125 \mathrm{MHz}, \mathrm{D}_{2} \mathrm{O}\right) \delta 102.62,84.00,81.75,78.18,73.84,73.68,73.08$ (overlap of 1C), 71.23, 71.03, 69.17, 50.98 (overlap of 3C), 31.33, 28.99, 28.66 (overlap of 3C), 28.58, 27.79, 25.47, 22.25, 21.23 (overlap of 2C), 21.16, 13.66. ESI for $\mathrm{C}_{30} \mathrm{H}_{56} \mathrm{O}_{18} \mathrm{~S}_{4} \mathrm{Na}_{4}[\mathrm{M}+\mathrm{Na}]^{+}$calcd. 947.18, found 947.10. DodGlu(PSNa) ${ }_{4}$ : yield $0.71 \mathrm{~g}, 0.76 \mathrm{mmol}, 44 \% ;{ }^{1} \mathrm{H}$ NMR(500 MHz, $\left.\mathrm{D}_{2} \mathrm{O}\right) \delta \quad 4.41$ $(\mathrm{d}, 1 \mathrm{H}), 3.92-3.68(\mathrm{~m}, 12 \mathrm{H}), 3.50(\mathrm{~m}, 1 \mathrm{H}), 3.42(\mathrm{~m}, 1 \mathrm{H}), 3.38(\mathrm{~m}, 1 \mathrm{H}), 3.15(\mathrm{t}, 1 \mathrm{H}), 3.00(\mathrm{~m}, 8 \mathrm{H})$, $2.03(\mathrm{~m}, 8 \mathrm{H}), 1.62(\mathrm{~m}, 2 \mathrm{H}), 1.29(\mathrm{~m}, 18 \mathrm{H}), 0.87(\mathrm{~m}, 3 \mathrm{H}) .{ }^{13} \mathrm{C} \mathrm{NMR}\left(125 \mathrm{MHz}, \mathrm{D}_{2} \mathrm{O}\right) \delta 102.58$, 84.11, 81.78, 78.09, 73.78, 72.33, 71.79, 71.76, 71.04, 69.81, 69.19, 48.38, 48.23, 48.14 (overlap of 1C), 31.53, 29.10, 29.03, 28.95, 28.90, 28.80, 28.58, 25.42 (overlap of 3C), 25.32, 24.62, 22.36, 13.75. ESI for $\mathrm{C}_{30} \mathrm{H}_{56} \mathrm{O}_{18} \mathrm{~S}_{4} \mathrm{Na}_{4}$ [M-Na] calcd. 901.20, found 901.10. NpBrGlu(PSNa) 4 : yield $0.92 \mathrm{~g}, 0.95 \mathrm{mmol}, 81 \%$; ${ }^{1} \mathrm{H}$ NMR(500 MHz, $\left.\mathrm{D}_{2} \mathrm{O}\right) \delta \quad 8.10(\mathrm{~d}, 1 \mathrm{H}), 7.86(\mathrm{~d}, 1 \mathrm{H}), 7.78(\mathrm{~d}, 1 \mathrm{H}), 7.66$ (dd, 1H), $7.35(\mathrm{~m}, 2 \mathrm{H}), 4.93(\mathrm{~d}, 1 \mathrm{H}), 4.03(\mathrm{~m}, 1 \mathrm{H}), 3.96(\mathrm{~m}, 3 \mathrm{H}), 3.84-3.79(\mathrm{~m}, 2 \mathrm{H}), 3.68-3.59$ $(\mathrm{m}, 4 \mathrm{H}), 3.46-3.38(\mathrm{~m}, 4 \mathrm{H}), 3.08-2.98(\mathrm{~m}, 6 \mathrm{H}), 2.92(\mathrm{~m}, 2 \mathrm{H}), 2.15-1.99(\mathrm{~m}, 8 \mathrm{H}) .{ }^{13} \mathrm{C} \mathrm{NMR}$ (125 MHz, $\left.\mathrm{D}_{2} \mathrm{O}\right) \delta 154.65,132.64,130.92,130.24,129.98,129.43,129.35,119.65,118.08,110.59$, $100.00,83.90,81.11,77.69,74.00,72.40,71.66,71.59,69.81,69.14,48.54,48.37,48.25$ (overlap of 1C), 25.45 (overlap of 2C), 24.73. ESI for $\mathrm{C}_{28} \mathrm{H}_{37} \mathrm{O}_{18} \mathrm{~S}_{4} \mathrm{Na}_{4} \mathrm{Br}^{81}$ [M-Na] calcd. 984.96, found 984.80. NpBrGlu(BuSNa) 4 : yield 1.00g, $0.98 \mathrm{mmol}, 73 \% ;{ }^{1} \mathrm{H} \mathrm{NMR}\left(500 \mathrm{MHz}, \mathrm{D}_{2} \mathrm{O}\right) \delta \quad 7.99$ (s, 1H), $7.74(\mathrm{~d}, 1 \mathrm{H}), 7.68(\mathrm{~d}, 1 \mathrm{H}), 7.59(\mathrm{~m}, 1 \mathrm{H}), 7.26(\mathrm{~m}, 2 \mathrm{H}), 4.93(\mathrm{~d}, 1 \mathrm{H}), 3.95(\mathrm{~m}, 1 \mathrm{H}), 3.84-3.74$ (m, 5H), $3.65(\mathrm{~m}, 1 \mathrm{H}), 3.53(\mathrm{~m}, 3 \mathrm{H}), 3.45-3.37(\mathrm{~m}, 4 \mathrm{H}), 2.99(\mathrm{~m}, 4 \mathrm{H}), 2.92(\mathrm{~m}, 2 \mathrm{H}), 2.78(\mathrm{~m}, 2 \mathrm{H})$, 
$1.89-1.60(\mathrm{~m}, 16 \mathrm{H}) .{ }^{13} \mathrm{C}$ NMR $\left(125 \mathrm{MHz}, \mathrm{D}_{2} \mathrm{O}\right) \delta 154.66,132.62,130.92,130.04,129.87$,

$129.34,129.24,119.73,118.01,110.94,100.28,83.74,81.34,77.87,74.17,73.62,73.03,72.84$,

$71.06,69.11,51.05$ (overlap of 2C), 50.87, 28.80, 28.74, 28.65, 27.95, 21.31 (overlap of 2C),

21.11. ESI for $\mathrm{C}_{32} \mathrm{H}_{45} \mathrm{O}_{18} \mathrm{~S}_{4} \mathrm{Na}_{4} \mathrm{Br}^{81}$ [M-Na] calcd. 995.00, found 994.90. PhGlu(PSNa) 4 : yield 0.80g, $0.96 \mathrm{mmol}, 65 \%$; ${ }^{1} \mathrm{H} \mathrm{NMR}\left(500 \mathrm{MHz}, \mathrm{D}_{2} \mathrm{O}\right) \delta 7.46(\mathrm{t}, 2 \mathrm{H}), 7.19(\mathrm{~m}, 3 \mathrm{H}), 5.19(\mathrm{~d}, 1 \mathrm{H}), 4.08$ $(\mathrm{m}, 1 \mathrm{H}), 4.00(\mathrm{~m}, 4 \mathrm{H}), 3.98(\mathrm{~m}, 1 \mathrm{H}), 3.86(\mathrm{~m}, 2 \mathrm{H}), 3.73(\mathrm{~m}, 2 \mathrm{H}), 3.63(\mathrm{~m}, 2 \mathrm{H}), 3.55-3.48(\mathrm{~m}, 2 \mathrm{H})$, $3.04(\mathrm{~m}, 8 \mathrm{H}), 2.09(\mathrm{~m}, 8 \mathrm{H}) . \quad{ }^{13} \mathrm{C}$ NMR $\left(125 \mathrm{MHz}, \mathrm{D}_{2} \mathrm{O}\right) \delta 156.61,130.23,123.58,116.63,100.16$, 83.95, 81.43, 77.98, 73.90, 72.32, 71.94, 71.77, 69.71, 69.09, 48.31, 48.25 (overlap of 1C), 48.12, 25.45, 25.32 (overlap of 1C), 24.66. ESI for $\mathrm{C}_{24} \mathrm{H}_{36} \mathrm{O}_{18} \mathrm{~S}_{4} \mathrm{Na}_{4}[\mathrm{M}+\mathrm{Na}]^{+}$calcd. 855.00, found 854.80. PhGlu(BuSNa) $)_{4}$ yield 0.76g, $0.86 \mathrm{mmol}, 68 \% ;{ }^{1} \mathrm{H}$ NMR(500 MHz, $\left.\mathrm{D}_{2} \mathrm{O}\right) \delta 7.47$ (t, 2H), $7.19(\mathrm{~m}$, 3H), 5.18(d, 1H), $4.03(\mathrm{~m}, 1 \mathrm{H}), 3.93(\mathrm{~m}, 4 \mathrm{H}), 3.85(\mathrm{~m}, 1 \mathrm{H}), 3.74(\mathrm{~m}, 3 \mathrm{H}), 3.64(\mathrm{~m}, 1 \mathrm{H}), 3.59(\mathrm{~m}$, 2H), $3.50-3.46(\mathrm{~m}, 2 \mathrm{H}), 2.99(\mathrm{~m}, 8 \mathrm{H}), 1.80(\mathrm{~m}, 16 \mathrm{H}) .{ }^{13} \mathrm{C} \mathrm{NMR}\left(125 \mathrm{MHz}, \mathrm{D}_{2} \mathrm{O}\right) \delta 156.61$, $130.19,123.60,116.75,100.37,83.83,81.44,77.96,74.04,73.68,73.12$ (overlap of 1C), 71.0, $69.03,50.98,50.90$ (overlap of 2C), 28.69, 28.51 (overlap of 1C), 27.84, 21.23 (overlap of 1C), 21.13, 21.09. ESI for $\mathrm{C}_{28} \mathrm{H}_{44} \mathrm{O}_{18} \mathrm{~S}_{4} \mathrm{Na}_{4}[\mathrm{M}-\mathrm{Na}]^{-}$calcd. 865.11, found 865.00. PhGal(PSNa) $)_{4}$ : yield 0.65g, $0.78 \mathrm{mmol}, 53 \% ;{ }^{1} \mathrm{H} \mathrm{NMR}\left(500 \mathrm{MHz}, \mathrm{D}_{2} \mathrm{O}\right) \delta 7.42(\mathrm{t}, 2 \mathrm{H}), 7.15(\mathrm{~d}, 3 \mathrm{H}), 5.11(\mathrm{~d}, 1 \mathrm{H}), 4.03-$ $3.61(\mathrm{~m}, 14 \mathrm{H}), 3.01(\mathrm{~m}, 8 \mathrm{H}), 2.05(\mathrm{~m}, 8 \mathrm{H}) . \quad{ }^{13} \mathrm{C}$ NMR $\left(125 \mathrm{MHz}, \mathrm{D}_{2} \mathrm{O}\right) \delta 157.26,130.60,123.88$, $117.34,101.22,81.93,79.30,75.44,74.37,72.49,72.29,70.21,70.08,69.77,48.84$ (overlap of 2C), 48.64, 25.44, 25.72, 25.13 (overlap of 1C). ESI for $\mathrm{C}_{24} \mathrm{H}_{36} \mathrm{O}_{18} \mathrm{~S}_{4} \mathrm{Na}_{4}$ [M-Na] calcd. 809.05, found 809.00. PhGal(SO S $\left._{3}\right)_{4}$ : yield $0.43 \mathrm{~g}, 0.64 \mathrm{mmol}, 32 \% ;{ }^{1} \mathrm{H} \mathrm{NMR}\left(500 \mathrm{MHz}, \mathrm{D}_{2} \mathrm{O}\right) \delta 7.43(\mathrm{~m}, 2 \mathrm{H})$, $7.21(\mathrm{~m}, 3 \mathrm{H}), 5.38(\mathrm{dd}, 1 \mathrm{H}), 5.16(\mathrm{~s}, 1 \mathrm{H}), 4.69(\mathrm{dd}, 2 \mathrm{H}), 4.38(\mathrm{~m}, 2 \mathrm{H}), 4.28(\mathrm{~m}, 1 \mathrm{H}) .{ }^{13} \mathrm{C} \mathrm{NMR}$ $\left(125 \mathrm{MHz}, \mathrm{D}_{2} \mathrm{O}\right) \delta 156.93,130.08,123.60,117.05,99.52,75.54,75.51,74.83,72.49,67.71$. ESI for $\mathrm{C}_{12} \mathrm{H}_{12} \mathrm{O}_{18} \mathrm{~S}_{4} \mathrm{Na}_{4}[\mathrm{M}+\mathrm{Na}]^{+}$calcd. 686.84, found 686.80 . 


\section{Reference:}

1. Park, O. J.; Kim, D. Y.; Dordick, J. S. Enzyme-catalyzed synthesis of sugar-containing monomers and linear polymers. Biotechnol. Bioeng. 2000, 70, 208.

2. Raku, T.; Kitagawa, M.; Shimakawa, H.; Tokiwa, Y. Enzymatic synthesis of trehalose esters having lipophilicity. J. Biotechnol. 2003, 100, 203.

3. Sun, X. L.; Grande, D.; Baskaran, S.; Hanson, S. R.; Chaikof, E. L. Glycosaminoglycan mimetic biomaterials. 4. Synthesis of sulfated lactose-based glycopolymers that exhibit anticoagulant activity. Biomacromolecules 2002, 3, 1065.

4. Michaeli, D. Pharmaceutical compositions containing synthetic polysulfated oligosaccharides for the enhancement of wound healing. Eur. Pat. Appl. 1987. 